\title{
Overexpression of miR-574-3p suppresses proliferation and induces apoptosis of chronic myeloid leukemia cells via targeting IL6/JAK/STAT3 pathway
}

\author{
HAOYING YANG ${ }^{1}$, JUN ZHANG $^{2}$, JIUPING LI ${ }^{1}$, FURONG ZHAO $^{1}$, YAO SHEN $^{2}$ and XUEMEI XING ${ }^{2}$
}

Departments of ${ }^{1}$ Blood Transfusion and ${ }^{2}$ Clinical Laboratory, San Er Ling Yi Hospital Affiliated to School of Medicine, Xi'an Jiaotong University, Hanzhong, Shanxi 723000, P.R. China

Received April 3, 2017; Accepted August 23, 2018

DOI: $10.3892 /$ etm.2018.6700

\begin{abstract}
The present study aimed to elucidate the potential roles and regulatory mechanism of microRNA (miR)-574-3p in the development of chronic myeloid leukemia (CML). The expression of miR-574-3p in peripheral blood obtained from patients with CML was examined. Subsequently, miR-574-3p was overexpressed and suppressed in CML K562 cells to further investigate the effects of miR-574-3p on cell proliferation, and apoptosis. Furthermore, a luciferase reporter assay was performed to investigate whether interleukin-6 (IL-6) was a target of miR-574-3p. In addition, the regulatory association between miR-574-3p and the IL-6/Janus kinase (JNK)/signal transducer and activator of transcription-3 (STAT3) signaling pathway was explored. The expression of miR-574-3p in the peripheral blood obtained from patients with CML was significantly lower compared with that in healthy controls. Overexpression of miR-574-3p significantly inhibited the proliferation and induced the apoptosis of K562 cells, whereas suppression of miR-574-3p exhibited opposite effects. In addition, IL-6 was identified to be a direct target of miR-574-3p. Overexpression of IL-6 significantly promoted the proliferation and inhibited the apoptosis of K562 cells. Furthermore, overexpression of miR-574-3p inhibited the activation of the JAK/STAT3 signaling pathway, which was rescued by overexpression of IL- 6 . The results of the current study indicate that miR-574-3p overexpression may serve an important role in inhibiting proliferation and inducing apoptosis of K562 cells via suppression of IL-6/JAK/STAT3 signaling pathway activation. miR-574-3p may serve as a potential therapeutic target for CML.
\end{abstract}

Correspondence to: Professor Xuemei Xing, Department of Clinical Laboratory, San Er Ling Yi Hospital Affiliated to School of Medicine, Xi'an Jiaotong University, No. 783 Tianhan Road, Hanzhong, Shanxi 723000, P.R. China

E-mail: xuemeixing23@126.com

Key words: chronic myeloid leukemia, miR-574-3p, IL6/JAK/STAT3 pathway, proliferation, apoptosis

\section{Introduction}

Chronic myeloid leukemia (CML) is a malignant myeloproliferative disease characterized by an excessive and unregulated production of myeloid leukemia cells in the bone marrow $(1,2)$, accounting for approximately $15 \%$ of newly diagnosed cases of leukemia in adults (3). Furthermore, the treatment of CML is problematic and its pathogenesis remains elusive (4). Therefore, it is important to elucidate the key mechanism underlying CML for the development of effective therapeutic strategies.

MicroRNAs (miRNAs) are small non-coding RNA recently implicated in numerous biological processes through via regulating gene expression (5-7). Accumulating studies have reported the aberrant expression and key roles of several miRNAs in CML (8-12). Exploration of key miRNAs associated with CML development has become a hot topic of research. Recently, the critical functions of miR-574-3p in numerous cancers have been illustrated. For instance, miR-574-3p was down-regulated in bladder cancer cells and overexpression of miR-574-3p significantly inhibits bladder cancer cell proliferation, migration and invasion (13). miR-574-3p is identified as potential novel prognostic marker for breast cancer by next generation sequencing profiling (14), which is also able to modulate tamoxifen response in breast cancer (15). Reduced expression of miR-574-3p is observed in the early stages of gastric cancer and overexpression of miR-574-3p inhibits proliferation, migration and invasion of gastric cancer SGC7901 cells in vitro (16). Nevertheless, the roles of miR-574-3p in regulating the development of CML remain unclear.

In the present study, the expression of miR-574-3p in peripheral blood obtained from CML patients was detected. Then miR-574-3p was overexpressed and suppressed in CML K562 cells to further investigate the effects of miR-574-3p on cell proliferation and apoptosis. Moreover, luciferase reporter assay was performed to investigate whether interleukin-6 (IL6) was a target of miR-574-3p. Besides, it is reported that IL6 can regulate the activation of JAK/STAT3 pathway in CML development (17), thus the regulatory relationship between miR-574-3p and IL6/Janus kinase/signal transducer and activator of transcription-3 (JAK/STAT3) pathway was 
explored. All efforts of our study were to elucidate the potential roles and regulatory mechanism of miR-574-3p in CML development.

\section{Materials and methods}

Patient samples. This study was approved by the Ethics Committee of San Er Ling Yi Hospital Affiliated to School of Medicine, Xi'an Jiaotong University, and each patient provided written informed consent. A total of 36 consecutive, treatment naive patients who were diagnosed with CML in our hospital and 8 healthy volunteers were enrolled in our study. Peripheral blood was then obtained from CML patients and healthy volunteers and the serum was collected by centrifugation at $1,500 \mathrm{~g}$ for $10 \mathrm{~min}$ at $4^{\circ} \mathrm{C}$. Finally, serum were portioned in aliquots and stored at $80^{\circ} \mathrm{C}$ until subsequently use.

Cell culture and transfection. Human CML cell line K562 (American Type Culture Collection, Manassas, VA, USA) was cultured in Roswell Park Memorial Institute (RPMI)-1640 medium (HyClone, Logan, UT, USA) supplemented with $10 \%$ fetal bovine serum (FBS, Sigma, USA) in a $5 \% \mathrm{CO}_{2}$ humidified incubator at $37^{\circ} \mathrm{C}$.

The miR-574-3p mimics, miR-574-3p inhibitor or their corresponding controls were synthesized by Shanghai GenePharma Co., Ltd. (Shanghai, China). Vector pcDNA-IL6 was constructed by inserting the coding oligonucleotides of IL6 into pcDNA3.1 vector (Invitrogen, Shanghai, China) for overexpression of IL6. Small interference RNAs (siRNAs) targeting IL6 (si-IL6) and corresponding control siRNAs (si-control) were designed and synthesized by RiboBio (Gungdong, China). For cell transfection, K562 cells $\left(6 \times 10^{4}\right.$ cells per well) were seeded in a 24 -well plate and then transfected with $50 \mathrm{nM}$ of miR-574-3p mimics or mimic control, miR-574-3p inhibitor or inhibitor control, pcDNA-IL6 or blank vector, and si-IL6 or si-control using Lipofectamine 2000 (Invitrogen, Shanghai, China) in accordance with the manufacturer's protocols. After $48 \mathrm{~h}$ of transfection, cells were harvested for subsequent analysis.

Dual luciferase reporter assay. Complementary oligonucleotides containing the miR-574-3p target site from IL6 (IL6 3'UTR-wt) and the mutated 3'UTR of IL6 (IL6 3'UTR-mut) containing a mutated miR-574-3p target sequence with identical flanking nucleotides were synthesized by Invitrogen (Shanghai, China). Oligonucleotides were then inserted into the 3'UTR of the pMIR-REPORT luciferase reporter vector (Ambion, Austin, Texas, USA) to construct a luciferase reporter. pMIR-REPORT Beta-gal was used as the internal control. K562 cells were cultured into a 24-well plate and then cotransfected with miR-574-3p mimic or mimic control and pMIR 3'UTR clones or pMIR Beta-gal for $24 \mathrm{~h}$ using Lipofectamine 2000 (Invitrogen, Shanghai, China). The luciferase and $\mathrm{b}$-galactosidase activity were then quantified using Dual-Light System chemiluminescent reporter gene assay (Applied Biosystems, Foster City, CA, USA).

MTT assay. The cell viability of K562 cells were detected by the 3-(4, 5-dimethylthiazol-2-yl)-2,5-diphenyltetrazo-liumbromide (MTT) assay. After transfection, $6 \times 10^{4}$ cells were plated into 6-well plates. At 1,2,3 days after transfection, $10 \mathrm{mg} / \mathrm{ml}$ of MTT solution (Sigma, USA) was added into each well and cultured the cells for $12 \mathrm{~h}$. Then $200 \mu \mathrm{l}$ of dimethyl sulfoxide (DMSO, Sigma, USA) was added into each well for $20 \mathrm{~min}$ to dissolve the formazan crystals. The absorbance at $490 \mathrm{~nm}$ was finally detected.

Colony formation assay. The colony formation ability of K562 cells was also detected. K562 cells in each group were diluted to 100 cells, seeded in soft agar plates and cultured in complete medium for 12 days. When the cells in the largest dilution formed clones, clones were then fixed with $100 \%$ methanol for $15 \mathrm{~min}$. After that, cells were stained with Giemsa (Sigma, USA) for $15 \mathrm{~min}$. The number of clones in 5 regions in each plate which were randomly selected was counted under a microscope (IX83, Olympus). Three parallel experiments were conducted for each sample.

Detection of cell apoptosis using flow cytometry. The apoptosis of K562 cells was measured using Annexin V labeling BD Annexin V-fluorescein isothiocyanate (FITC) assay kit (Becton Dickinson, NJ, USA). In brief, a total of $5 \times 10^{5}$ cells were harvested after transfection of $48 \mathrm{~h}$. Cells were stained with $5 \mu \mathrm{l}$ Annexin V-FITC and propidium iodide (PI) in the dark for $15 \mathrm{~min}$. Within $1 \mathrm{~h}$, the apoptotic cells were sent out by means of Flow BD Fac Canto II flow-cytometer (Becton Dickinson, NJ, USA). According to the fluorescence intensity, the apoptotic cells (annexin V-positive and PI-negative) were finally analyzed with a CellQuest 3.0 software (Becton Dickinson, NJ, USA).

RNA extraction and quantitative real-time PCR ( $q R T-P C R)$. Total RNA was extracted from peripheral blood and K562 cells using Trizol reagent (Invitrogen, Shanghai, China) and their quality was measured using ultraviolet spectrophotometer (SMA 400 UV-VIS, Merinton, Shanghai, China). First-strand cDNA was then synthesized using a High Capacity cDNA Reverse Transcription Kit (Invitrogen, Shanghai, China). To detect the expressions of miR-574-3p and IL6, qRT-PCR was performed using the SYBR ExScript RT-qPCR Kit (Takara, China) by means of the ABI PRISM 7300 Fast Real-Time PCR System (Ambion, Foster City, CA, USA). Moreover, analysis of melting curve was conducted at the end of each PCR to detect the specificity of the PCR products. U6 and Phosphoglyceraldehyde dehydrogenase (GAPDH) was used as the internal control for miR-574-3p and IL6, respectively. Finally, the relative expression of miR-574-3p and IL6 was calculated with $2^{-\Delta \Delta \mathrm{Ct}}$ method.

Enzyme-linkedimmunosorbentassay (ELISA) assay. Peripheral blood $(2 \mathrm{ml})$ was then obtained from CML patients and healthy volunteers and the serum was collected by centrifugation at $350 \mathrm{~g}$ for $10 \mathrm{~min}$. The concentration of IL6 was then determined with a commercially available ELISA kit (Genzyme, Boston, MA) according to the manufacturer's protocols.

Western blot analysis. Total proteins in whole-cell lysates of K562 cells were obtained using radioimmunoprecipitation buffer (RIPA) (Sangon Biotech, Shanghai, China) containing protease inhibitor cocktail (GE Healthcare, Buckinghamshire, UK) on ice for $20 \mathrm{~min}$. After estimating the protein 
concentration by Bradford reagent (Biorad laboratories, CA, USA), equal quantities of protein extracts were separated in a $10 \%$ SDS-PAGE and then electrically transferred to polyvinylidene difluoride membrane (Amersham, GE, Buckinghamshire, UK). After blocked with 5\% blocking agent, the membranes were immunoblotted with primary antibodies, including anti-IL6, anti-JAK1, anti-p-JAK1, anti-JAK2, anti-p-JAK2, anti-STAT3, anti-p-STAT3 and anti- $\beta$-actin $(1: 1,000$, cell signaling, MA, USA) overnight at $4^{\circ} \mathrm{C}$ in shaker. $\beta$-actin was used as the loading control. The membranes were then incubated with secondary horseradish peroxidase-conjugated antibody (1:5,000, cell signaling, MA, USA) for $1 \mathrm{~h}$. Lastly, the membranes were visualized with Amersham ECL Detection Agent (GE, Buckinghamshire, UK) by exposure to hyperfilm (GE, Buckinghamshire, UK) in the dark room.

Statistical analysis. Data were presented as mean \pm standard deviation (SD) and their normal distribution was conducted using one-sample K-S test. Significant differences between groups were assessed using Student's t-test. All statistical analyses were performed using SPSS 18.0 (SPSS, Chicago, IL) and $\mathrm{P}<0.05$ was considered statistical significant.

\section{Results}

miR-574-3p was down-regulated in peripheral blood obtained from CML patients. As shown in Fig. 1A, the expression of miR-574-3p in peripheral blood obtained from CML patients was significantly lower than that in healthy controls $(\mathrm{P}<0.05)$, indicating miR-574-3p may be involved in the development and progression of CML. Overexpression of miR-574-3p inhibited proliferation and induced apoptosis of K562 cells.

To investigate the potential effects of miR-574-3p in CML, K562 cells were transfected with miR-574-3p mimic, mimic control, miR-574-3p inhibitor and inhibitor control. The results of MTT assay showed that after 2 and 3 days of transfection, miR-574-3p mimics significantly inhibited cell viability while miR-574-3p inhibitor markedly enhanced cell viability when compared with their corresponding controls $(\mathrm{P}<0.05$, Fig. 1B). In comparison with their corresponding control groups, miR-574-3p expression was significantly increased in miR-574-3p mimic group, while obviously decreased in miR-574-3p inhibitor group after 2 days of transfection $(\mathrm{P}<0.05$, Fig. 1C). Thus, colony formation assay and flow cytometry were further performed the cell proliferation and apoptosis after 2 days of transfection. The results showed miR-574-3p mimics decreased the number of colony $(\mathrm{P}<0.05$, Fig. 1D) and induced cell apoptosis ( $\mathrm{P}<0.05$, Fig. 1E) significantly compared with mimic control. Moreover, miR-574-3p inhibitor resulted in opposite effects on colony number and cell apoptosis (Fig. 1D and E).

IL6 was the direct target of miR-574-3p. According to the information of TargetScanHuman, IL6 was the predicted as a potential target of miR-574-3p. Luciferase report assay was performed to further verify the predicted results. Expected results was obtained that miR-574-3p mimic significantly inhibited the luciferase report activity of IL6 3'UTR-wt rather than IL6 3'UTR-mut ( $\mathrm{P}<0.05$, Fig. 2A). Then qRT-PCR and western blot were performed to detect the expression of IL6 in different transfected groups. The results showed the expression of IL6 in both mRNA and protein levels was significantly inhibited in miR-574-3p mimic group compared with that in mimic control group, while was markedly increased in miR-574-3p inhibitor group compared with that in inhibitor control group $(\mathrm{P}<0.05$, Fig. $2 \mathrm{~B}$ and $\mathrm{C})$. Notably, the mRNA expression of IL6 in peripheral blood obtained from CML patients and healthy controls was detected. Expected results were obtained that IL6 expression was significantly higher than that in healthy controls $(\mathrm{P}<0.05$, Fig. 2D). ELISA assay also showed similar results that the concentration of IL6 was significantly higher than that in healthy controls $(\mathrm{P}<0.05$, Fig. 2E). These data indicated that IL6 was the direct target of miR-574-3p and its expression was negatively regulated by miR-574-3p.

Overexpression of IL6 promoted proliferation and inhibited apoptosis of $\mathrm{K562}$ cells. We further investigated whether dys-regulation of IL6 could also regulate K562 cell proliferation and apoptosis. Thus, K562 cells were transfected with pcDNA-IL6, blank vector, si-IL6 and si-control. As shown in Fig. 3A, when compared with their corresponding controls, overexpression of IL6 could significantly increase K562 cell viability after 2 and 3 days of pcDNA-IL6 transfection, while knockdown of IL6 resulted in a significantly decrease of cell viability after 2 and 3 days of si-IL6 transfection $(\mathrm{P}<0.05)$. In addition, after 2 days of transfection, the protein expression of IL6 was significantly up-regulated in pcDNA-IL6 group and obvious down-regulated in si-IL6 group in comparison with their corresponding control groups $(\mathrm{P}<0.05$, Fig. 3B). Besides, the results of colony formation assay and flow cytometry showed, after 2 days of pcDNA-IL6 transfection, the number of colony was significantly increased $(\mathrm{P}<0.05$, Fig. $3 \mathrm{C})$ and apoptotic cells were markedly decreased ( $\mathrm{P}<0.05$, Fig. 3D). However, opposite effects were observed after 2 days of si-IL6 transfection (Fig. 1D and E). These data indicated that overexpression of IL6 promoted proliferation and inhibited apoptosis of K562 cells, which was opposite with the function of miR-574-3p overexpression.

miR-574-3p targets IL6 to further inhibit the activation of JAK/STAT3 pathway in K562 cells. We further investigated the relationship between miR-574-3p and IL6/JAK/STAT3 pathway, aiming to elucidate the key mechanism of miR-574-3p in CMI development. The results showed that the protein levels of IL6, p-JAK1/JAK1, p-JAK2/JAK2 and p-STAT3/STAT3 in miR-574-3p mimic group were all significantly lower than that in mimic control group or untransfected group $(\mathrm{P}<0.05$, Fig. 4$)$, indicating that overexpression of miR-574-3p could inhibited the activation of JAK/STAT3 pathway. However, the protein levels of IL6, p-JAK1/JAK1, p-JAK2/JAK2 and p-STAT3/STAT3 were significantly increased in miR-574-3p mimic+pcDNA-IL6 group in comparison with that in miR-574-3p mimic group or miR-574-3p mimic+blank vector group $(\mathrm{P}<0.05$, Fig. 4$)$, indicating that overexpression of IL6 could reverse the inhibitory of miR-574-3p overexpression on the activation of IL6/JAK/STAT3 pathway, and miR-574-3p might inhibit the activation of JAK/STAT3 pathway in K562 cells via targeting IL6. 

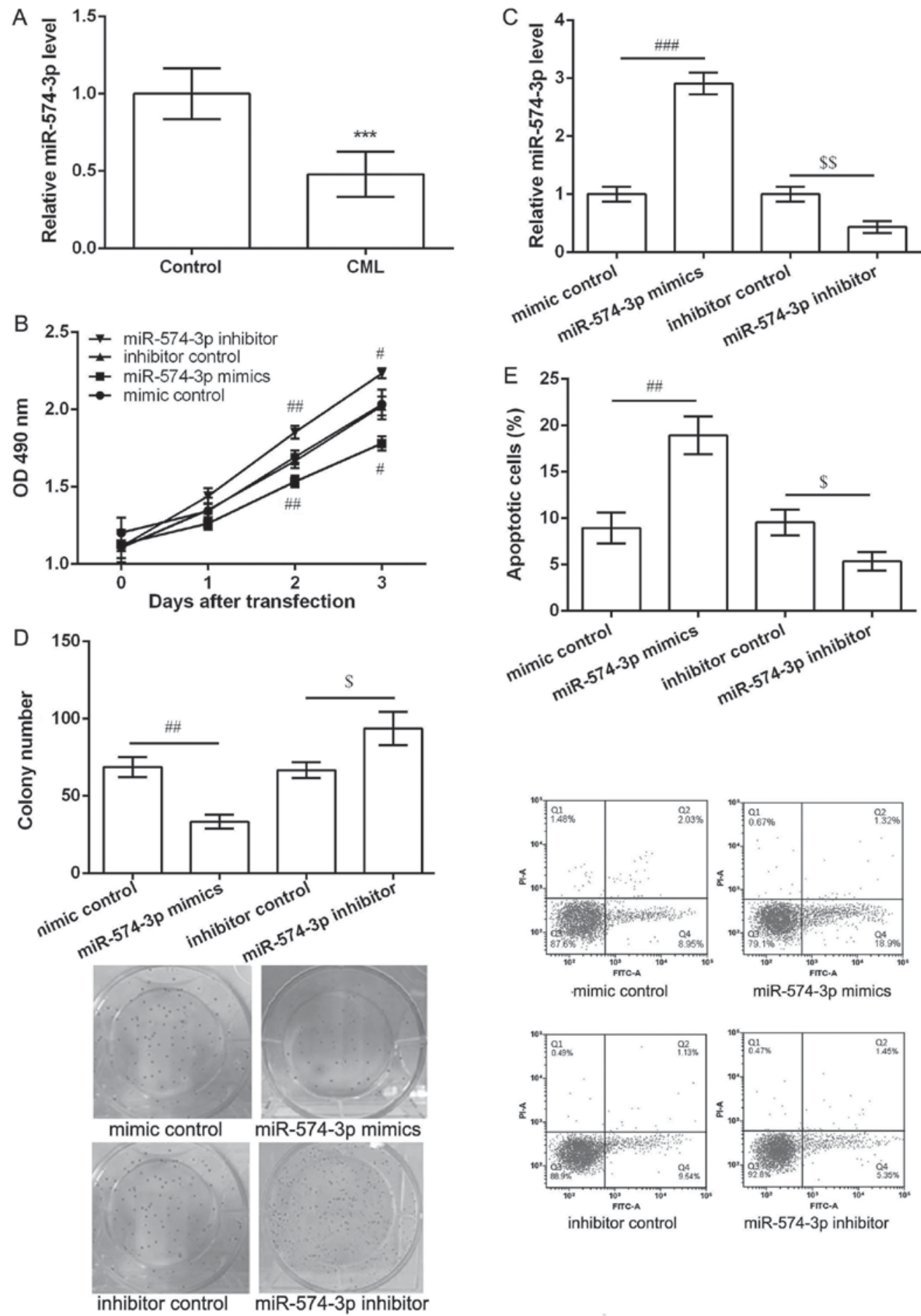

Figure 1. Expression of miR-574-3p in the peripheral blood of CML patients and the effects of miR-574-3p on the proliferation and apoptosis of CML K562 cells which were transfected with miR-574-3p mimic, mimic control, miR-574-3p inhibitor and inhibitor control. (A) The expression of miR-574-3p in the peripheral blood of CML patients and healthy controls. (B) MTT assay showed the cell viability in different transfected groups after different times of transfection. (C) The expression of miR-574-3p in different transfected groups after 2 days of transfection. (D) Colony formation assay showed the colony number in different transfected groups after 2 days of transfection. (E) Flow cytometry showed the apoptosis in different transfected groups after 2 days of transfection. Error bars indicate means $\pm \mathrm{SD}$. ${ }^{* * *} \mathrm{P}<0.001$ vs. control; ${ }^{\# \mathrm{P}}<0.05,{ }^{\# \#} \mathrm{P}<0.01$ and ${ }^{\# \# \#} \mathrm{P}<0.001$ vs. mimic control; ${ }^{\$ \mathrm{P}}<0.05$ and ${ }^{\$ \$} \mathrm{P}<0.01$ vs. inhibitor control.

\section{Discussion}

In the present study, miR-574-3p was down-regulated in peripheral blood obtained from CML patients, and down-regulation of miR-574-3p promoted proliferation and inhibited apoptosis of K562 cells. In addition, IL6 was confirmed as a direct target of miR-574-3p, and the effects of IL6 overexpression on K562 cell proliferation and apoptosis were opposite with down-regulation of miR-574-3p. Besides, the activation of JAK/STAT3 pathway induced by miR-574-3p overexpression was rescued by overexpression of IL6. These data imply that miR-574-3p may exert a tumor suppressor role in K562 cells via regulating IL6/JAK/STAT3 pathway. 
A

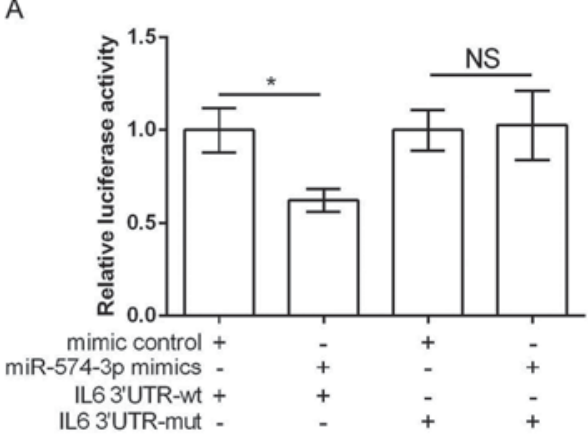

D

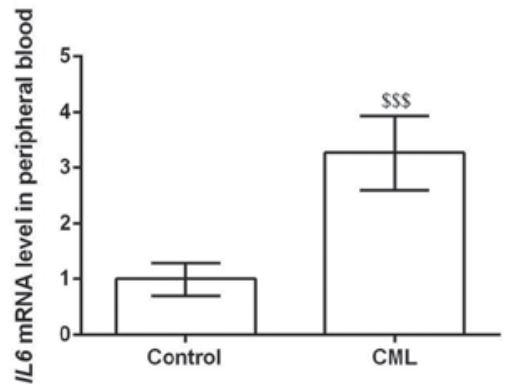

B

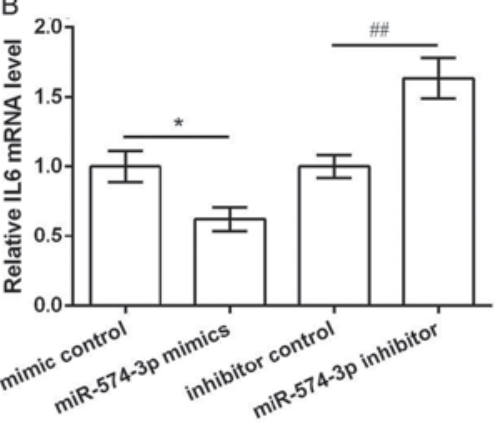

$\mathrm{E}$

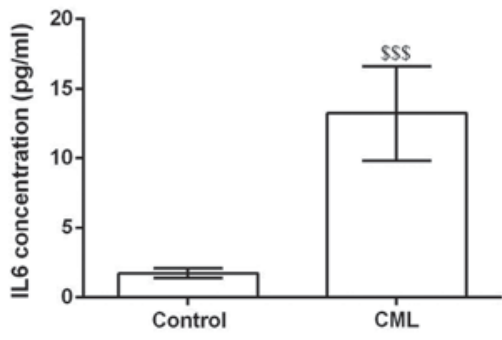

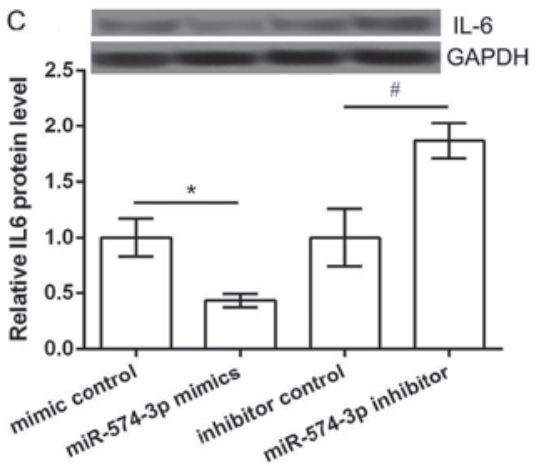

Figure 2. IL6 was the direct target of miR-574-3p. (A) Luciferase report assay showed that miR-574-3p directly targeted the IL6 3'UTR-wt. (B) The mRNA expression of IL6 in different transfection groups determined by qRT-PCR. (C) The protein expression of IL6 in different transfection groups determined by western blot. (D) The mRNA expression of IL6 in peripheral blood obtained from CML patients and healthy controls determined by qRT-PCR. (E) The concentration of IL6 in peripheral blood obtained from CML patients and healthy controls determined by ELISA. Error bars indicate means \pm SD. ${ }^{*} \mathrm{P}<0.05$ vs. mimic control. ${ }^{\#} \mathrm{P}<0.05$ and ${ }^{\# \#} \mathrm{P}<0.01$ vs. inhibitor control. ${ }^{\$ \$} \mathrm{P}<0.001$ vs. control.

A

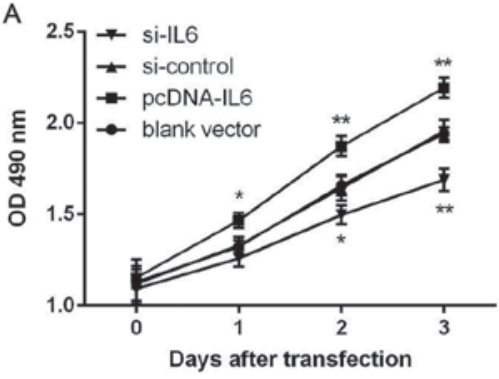

B
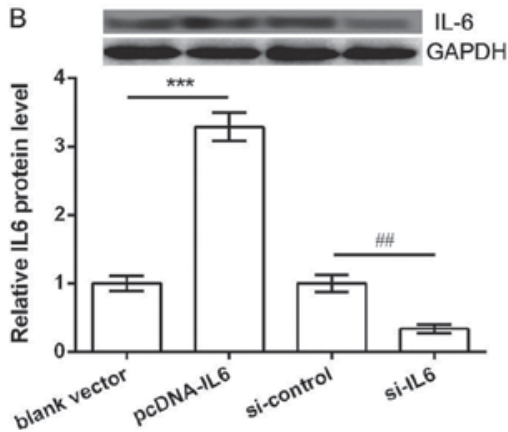

C
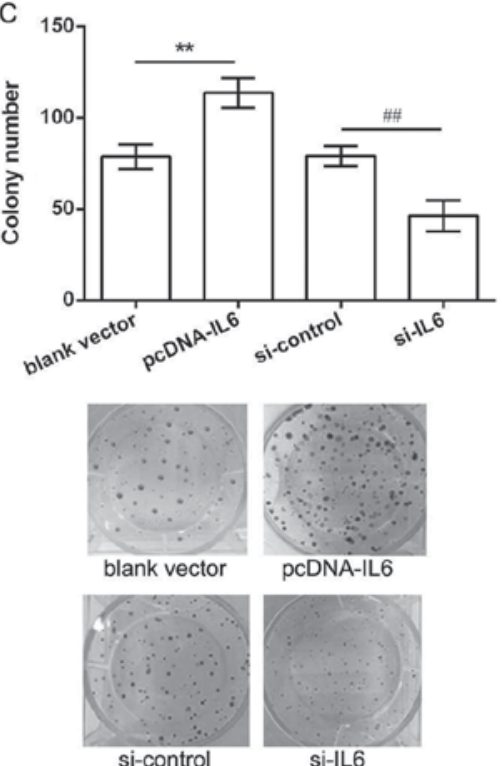
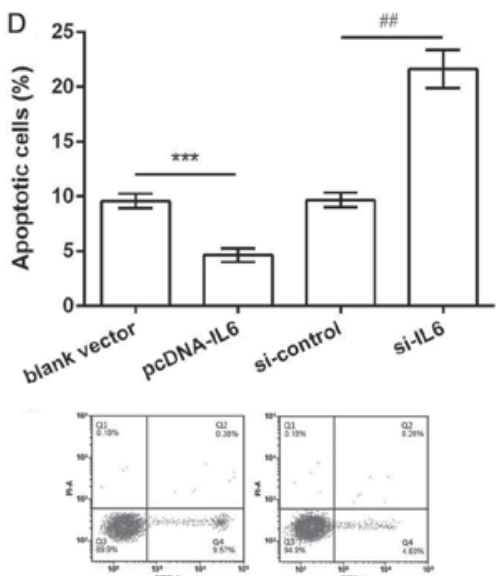

blank vector pcDNA-IL6

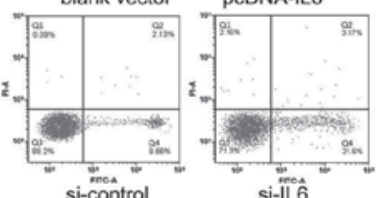

Figure 3. The effects of IL6 on the proliferation and apoptosis of CML K562 cells. K562 cells were transfected with pcDNA-IL6, blank vector, si-IL6 and si-control. (A) MTT assay showed the cell viability in different transfected groups after different times of transfection. The maximum absorption value is 490. (B) The expression of IL6 in different transfected groups after 2 days of transfection. (C) Colony formation assay showed the colony number in different transfected groups after 2 days of transfection. (D) Flow cytometry showed the apoptosis in different transfected groups after 2 days of transfection. Error bars indicate means $\pm \mathrm{SD} .{ }^{*} \mathrm{P}<0.05,{ }^{* *} \mathrm{P}<0.01$ and ${ }^{* * *} \mathrm{P}<0.001$. vs. blank vector control. ${ }^{\# \#} \mathrm{P}<0.01$ vs. si-control.

In previous studies, miR-574-3p has a tumor-promoting role in human osteosarcoma (18), while exerts a tumor suppressor effect in esophageal squamous cell carcinoma (19), indicating that miR-574-3p may play a dual role in regulating cancer progression. Furthermore, it is reported that aberrantly expression of miR-574-3p may play functional roles in regulating the proliferation and metatasis of gastric cancer (16). In our study, miR-574-3p was down-regulated in peripheral blood obtained from CML patients, and overexpression of miR-574-3p significantly inhibited proliferation and induced 

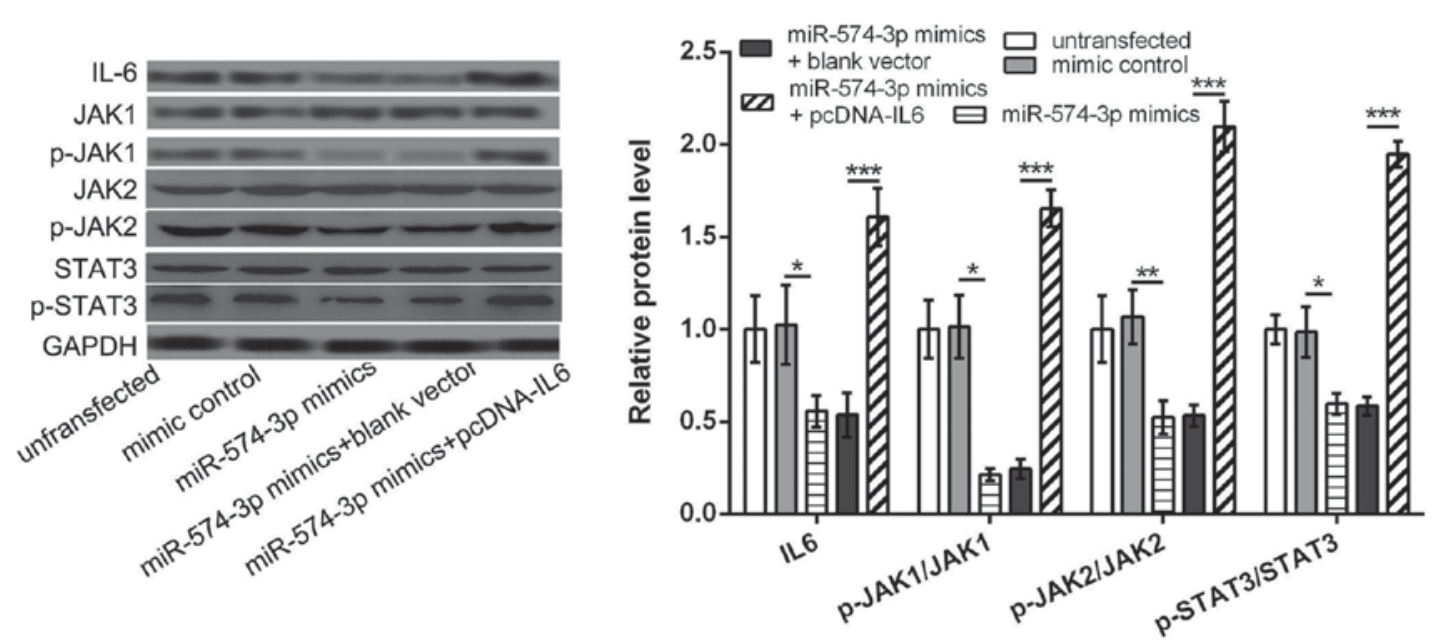

Figure 4. The protein expression of IL6, p-JAK1, JAK1, p-JAK2, JAK2, p-STAT3 and STAT3 in different transfected groups. Error bars indicate means \pm SD ${ }^{*} \mathrm{P}<0.05,{ }^{* *} \mathrm{P}<0.01$ and ${ }^{* * *} \mathrm{P}<0.001$.

apoptosis of K562 cells, indicating that miR-574-3p may play a tumor suppressor role in CML development.

In addition, miRNAs are implicated in numerous biological processes through degrading or inhibiting the translation of their target genes (20). In our study, IL6 was confirmed as a direct target of miR-574-3p. The effects of overexpression of IL6 on the proliferation and apoptosis of K562 cells were opposite with overexpression of miR-574-3p. IL-6, a pro-inflammatory cytokine, is previously shown to play important roles in the pathogenesis of hematological malignancies, including multiple myeloma (21). Maeda et al (22) confirmed that IL6 was implicated in both myeloid expansion and lymphocytopenia. Reynaud et al (23) demonstrated that IL6 could control the cell fate of leukemic multipotent progenitor cells fate and might serves as a positive feedback loop to sustain CML development. IL-6 is also considered as a selective and prognostic factor to address the follow-up in imatinib-treated CML patients (24). Although the role of miR-574-3p in CML has not been fully investigated, our results prompt us to speculate that miR-574-3p may inhibit proliferation and induce apoptosis of K562 cells through targeting IL6.

Furthermore, JAK/STAT signaling pathway has been implicated in transmission of information carried by cytokines. It is reported that JAK/STAT3 pathway can be activated by IL6, thus contributes to the CML development (17). Kumar et al (25), also demonstrated that IL-6 can induce JAK/STAT and MAPK pathways, thus have a key role in deciphering the CML progression stages. In addition, STAT5 is found to play a critical role in regulating growth and apoptosis of the CML leukaemic cells (26). JAK/STAT3 pathway is shown to be a key mechanism to mediate cryptotanshinone-induced apoptosis of CML K562 cells (27). Besides, capsaicin can induce apoptosis and inhibit proliferation of K562 leukemic cells through affecting miR-520a-5p/STAT3 interaction (28). Bortezomib can induce apoptosis of K562 leukemic cells by interacting with JAK/STAT pathway (29). JAK/STAT signaling pathway is found to mediate sehydrocostus lactone-suppressed proliferation of CML cells (30). In this study, overexpression of miR-574-3p could inhibit the activation of JAK/STAT3 pathway, which was rescued by overexpression of IL6. Therefore, we speculate that miR-574-3p may exert a tumor suppressor role in K562 cells via regulating IL6/JAK/STAT3 pathway, which are still needed to be further investigated.

In conclusion, our results indicate that overexpression of miR-574-3p may inhibit proliferation and induce apoptosis of K562 cells via suppressing the activation of IL6/JAK/STAT3 pathway. miR-574-3p may serve as a potential target for the treatment of CML.

\section{Acknowledgements}

Not applicable.

\section{Funding}

No funding was received.

\section{Availability of data and materials}

The analyzed data sets generated during the present study are available from the corresponding author on reasonable request.

\section{Authors' contributions}

HY, JZ and XX conceived and designed the experiments. HY, FZ and YS performed the experiments. JL and FZ analyzed the data. HY and XX wrote and revised the manuscript. All authors read and approved the final manuscript.

\section{Ethics approval and consent to participate}

The present study was approved by the Ethics Committee of San Er Ling Yi Hospital Affiliated to School of Medicine, Xi'an Jiaotong University, and each patient provided written informed consent.

\section{Patient consent for publication}

Not applicable. 


\section{Competing interests}

The authors declare that they have no competing interests.

\section{References}

1. Aquino SS, Gonçalves RP and Silva LB: The pharmacotherapeutic follow-up of patients with chronic myeloid leukemia (CML) on imatinib mesylate therapy. Rev Bras Hematol Hemoter 31: 137-142, 2009.

2. Marzag H: D\&eacute; veloppement de nouvelles r\&eacute;actions \&eacute;co-compatibles: Application \&agrave; la synth\&egrave;se de mol\&eacute;cules bioactives, 2013.

3. Jabbour E and Kantarjian H: Chronic myeloid leukemia: 2014 update on diagnosis, monitoring, and management. Am J Hematol 89: 547-556, 2014.

4. Holyoake TL, Jiang X, Drummond MW, Eaves AC and Eaves CJ: Elucidating critical mechanisms of deregulated stem cell turnover in the chronic phase of chronic myeloid leukemia. Leukemia 16: 549-558, 2002.

5. Chu Y, Zhu H, Lv L, Zhou Y and Huo J: MiRNAs in oesophageal squamous cancer. Neth J Med 71: 69-75, 2013.

6. Mo MH, Chen L, Fu Y, Wang W and Fu SW: Cell-free circulating miRNA biomarkers in cancer. J Cancer 3: 432-448, 2012.

7. Reddy KB: MicroRNA (miRNA) in cancer. Cancer Cell Int 15 : 38, 2015.

8. Arya D, Sachithanandan SP, Ross C, Palakodeti D, Li S and Krishna S: MiRNA182 regulates percentage of myeloid and erythroid cells in chronic myeloid leukemia. Cell Death Dis 8: e2547, 2017.

9. Xishan Z, Ziying L, Jing D and Gang L: MicroRNA-320a acts as a tumor suppressor by targeting BCR/ABL oncogene in chronic myeloid leukemia. Sci Rep 5: 12460, 2015.

10. Liu Y, Song Y, Ma W, Zheng W and Yin H: Decreased microRNA-30a levels are associated with enhanced ABL1 and BCR-ABL1 expression in chronic myeloid leukemia. Leuk Res 37: 349-356, 2013.

11. Yu Y, Yang L, Zhao M, Zhu S, Kang R, Vernon P, Tang D and Cao L: Targeting microRNA-30a-mediated autophagy enhances imatinib activity against human chronic myeloid leukemia cells Leukemia 26: 1752-1760, 2012

12. Li Y, Zhao L, Li N, Miao Y, Zhou H and Jia L: miR-9 regulates the multidrug resistance of chronic myelogenous leukemia by targeting ABCB1. Oncol Rep 37: 2193-2200, 2017.

13. Tatarano S, Chiyomaru T, Kawakami K, Enokida H, Yoshino H, Hidaka H, Nohata N, Yamasaki T, Gotanda T, Tachiwada T, et al: Novel oncogenic function of mesoderm development candidate 1 and its regulation by MiR-574-3p in bladder cancer cell lines. Int J Oncol 40: 951-959, 2012.

14. Krishnan P, Ghosh S, Wang B, Li D, Narasimhan A, Berendt R, Graham K, Mackey JR, Kovalchuk O and Damaraju S: Next generation sequencing profiling identifies miR-574-3p and miR-660-5p as potential novel prognostic markers for breast cancer. BMC Genomics 16: 735, 2015.

15. Ujihira T, Ikeda K, Suzuki T, Yamaga R, Sato W, Horie-Inoue K, Shigekawa T, Osaki A, Saeki T, Okamoto K, et al: MicroRNA-574-3p, identified by microRNA library-based functional screening, modulates tamoxifen response in breast cancer. Sci Rep 5: 7641, 2015.
16. Su Y, Ni Z, Wang G, Cui J, Wei C, Wang J, Yang Q, Xu Y and Li F: Aberrant expression of microRNAs in gastric cancer and biological significance of miR-574-3p. Int Immunopharmacol 13: 468-475, 2012.

17. Ma L, Zhu Z, Jiang L, Sun X, Lu X, Zhou M, Qian S and Jianyong L: Matrine suppresses cell growth of human chronic myeloid leukemia cells via its inhibition of the interleukin-6/Janus activated kinase/signal transducer and activator of transcription 3 signaling cohort. Leuk Lymphoma 56: 2923-2930, 2015.

18. Xu H, Liu X, Zhou J, Chen X and Zhao J: miR-574-3p acts as a tumor promoter in osteosarcoma by targeting SMAD4 signaling pathway. Oncol Lett 12: 5247-5253, 2016.

19. Okumura T, Kojima H, Miwa T, Sekine S, Hashimoto I, Hojo S, Nagata T and Shimada Y: The expression of microRNA 574-3p as a predictor of postoperative outcome in patients with esophageal squamous cell carcinoma. World J Surg Oncol 14: 228, 2016.

20. Yamasaki T, Kim EJ, Cerutti $\mathrm{H}$ and Ohama T: Argonaute 3 is a key player in miRNA-mediated target cleavage and translational repression in Chlamydomonas. Plant J 85: 258-268, 2016.

21. Hong DS, Angelo LS and Kurzrock R: Interleukin-6 and its receptor in cancer: Implications for translational therapeutics. Cancer 110: 1911-1928, 2007.

22. Maeda K, Baba Y, Nagai Y, Miyazaki K, Malykhin A, Nakamura K, Kincade PW, Sakaguchi N and Coggeshall KM: IL-6 blocks a discrete early step in lymphopoiesis. Blood 106: $879-885,2005$

23. Reynaud D, Pietras E, Barry-Holson K, Mir A, Binnewies M, Jeanne M, Sala-Torra O, Radich JP and Passegué E: IL-6 controls leukemic multipotent progenitor cell fate and contributes to chronic myelogenous leukemia development. Cancer Cell 20: 661-673, 2011.

24. Ciarcia R, Vitiello MT, Galdiero M, Pacilio C, Iovane V, d'Angelo D, Pagnini D, Caparrotti G, Conti D, Tomei V, et al: Imatinib treatment inhibit IL-6, IL-8, NF-KB and AP-1 production and modulate intracellular calcium in CML patients. J Cell Physiol 227: 2798-2803, 2012.

25. Kumar H, Tichkule S, Raj U, Gupta S, Srivastava S and Varadwaj PK: Effect of STAT3 inhibitor in chronic myeloid leukemia associated signaling pathway: A mathematical modeling, simulation and systems biology study. 3 Biotech 6: 40, 2016.

26. Baśkiewicz-Masiuk $M$ and Machaliński B: The role of the STAT5 proteins in the proliferation and apoptosis of the CML and AML cells. Eur J Haematol 72: 420-429, 2004.

27. Jung JH, Kwon TR, Jeong SJ, Kim EO, Sohn EJ, Yun M and Kim SH: Apoptosis induced by tanshinone IIA and cryptotanshinone is mediated by distinct JAK/STAT3/5 and SHP1/2 signaling in chronic myeloid leukemia K562 cells. Evid Based Complement Alternat Med 2013: 805639, 2013.

28. Kaymaz BT, Cetintaş VB, Aktan C and Kosova B: MicroRNA-520a-5p displays a therapeutic effect upon chronic myelogenous leukemia cells by targeting STAT3 and enhances the anticarcinogenic role of capsaicin. Tumor Biol 35: 8733-8742, 2014.

29. Selvi N, Kaymaz BT, Gündüz C, Aktan Ç, Kiper HD, Şahin F, Cömert M, Selvi AF, Kosova B and Saydam G: Bortezomib induces apoptosis by interacting with JAK/STAT pathway in K562 leukemic cells. Tumor Biol 35: 7861-7870, 2014.

30. Cai H, Qin X and Yang C: Dehydrocostus lactone suppresses proliferation of human chronic myeloid leukemia cells through Bcr/Abl-JAK/STAT signaling pathways. J Cell Biochem 118: 3381-3390, 2017. 